\title{
List of definitions and notations
}

\section{Set theory}

$|M|$ is the cardinality of a set $M$ (if $G$ is a finite group, then $|G|$ is called its order).

$x \in M(x \notin M)$ means that $x$ is (is not) an element of a set $M . N \subseteq M(N \nsubseteq M)$ means that $N$ is (is not) a subset of the set $M$; moreover, if $M \neq N \subseteq M$ we write $N \subset M$.

$\varnothing$ is the empty set.

$N$ is called a nontrivial subset of $M$, if $N \neq \varnothing$ and $N \subset M$. If $N \subset M$ we say that $N$ is a proper subset of $M$.

$M \cap N$ is the intersection and $M \cup N$ is the union of sets $M$ and $N$. If $M, N$ are sets, then $N-M=\{x \in N \mid x \notin M\}$ is the difference of $N$ and $M$.

$\mathbb{Z}$ is the set (ring) of integers: $\mathbb{Z}=\{0, \pm 1, \pm 2, \ldots\}$.

$\mathbb{N}$ is the set of all natural numbers.

$\mathbb{Q}$ is the set (field) of all rational numbers.

$\mathbb{R}$ is the set (field) of all real numbers.

$\mathbb{C}$ is the set (field) of all complex numbers.

\section{Number theory and general algebra}

$p$ is always a prime number.

$\pi$ is a set of primes; $\pi^{\prime}$ is the set of all primes not contained in $\pi$.

$m, n, k, r, s$ are, as a rule, natural numbers.

$\pi(m)$ is the set of prime divisors of $m$; then $m$ is a $\pi$-number.

$n_{p}$ is the $p$-part of $n, n_{\pi}$ is the $\pi$-part of $n$.

$(m, n)$ is the greatest common divisor of $m$ and $n$.

$m \mid n$ should be read as: $m$ divides $n$.

$m \nmid n$ should be read as: $m$ does not divide $n$. 
$\mathrm{GF}\left(p^{m}\right)$ is the finite field containing $p^{m}$ elements.

$\mathbb{F}^{*}$ is the multiplicative group of a field $\mathbb{F}$.

$\mathscr{L}(G)$ is the lattice of all subgroups of a group $G$.

If $n=p_{1}^{\alpha_{1}} \ldots p_{k}^{\alpha_{k}}$ is the standard prime decomposition of $n$, then $\lambda(n)=\sum_{i=1}^{k} \alpha_{i}$.

\section{Groups}

We consider only finite groups which are denoted, with a pair exceptions, by upper case Latin letters.

If $G$ is a group, then $\pi(G)=\pi(|G|)$.

$G$ is a $p$-group if $|G|$ is a power of $p ; G$ is a $\pi$-group if $\pi(G) \subseteq \pi$.

$G$ is, as a rule, a finite $p$-group.

$H \leq G$ means that $H$ is a subgroup of $G$.

$H<G$ means that $H \leq G$ and $H \neq G$ (in that case $H$ is called a proper subgroup of $G) .\{1\}$ denotes the group containing only one element.

$H$ is a nontrivial subgroup of $G$ if $\{1\}<H<G$.

$H$ is a maximal subgroup of $G$ if $H<G$ and it follows from $H \leq M<G$ that $H=M$.

$H \unlhd G$ means that $H$ is a normal subgroup of $G$; moreover, if, in addition, $H \neq G$ we write $H \triangleleft G$ and say that $H$ is a proper normal subgroup of $G$. Expressions 'normal subgroup of $G$ ' and ' $G$-invariant subgroup' are synonyms.

$H \triangleleft G$ is called a nontrivial normal subgroup of $G$ provided $H>\{1\}$.

$H$ is a minimal normal subgroup of $G$ if (a) $H \unlhd G$; (b) $H>\{1\}$; (c) $N \triangleleft G$ and $N<H$ implies $N=\{1\}$. Thus, the group $\{1\}$ has no minimal normal subgroup.

$G$ is simple if it is a minimal normal subgroup of $G$ (so $|G|>1$ ).

$H$ is a maximal normal subgroup of $G$ if $H<G$ and $G / H$ is simple.

The subgroup generated by all minimal normal subgroups of $G$ is called the socle of $G$ and denoted by $\operatorname{Sc}(G)$. We put, by definition, $\operatorname{Sc}(\{1\})=\{1\}$.

$\mathrm{N}_{G}(M)=\left\{x \in G \mid x^{-1} M x=M\right\}$ is the normalizer of a subset $M$ in $G$.

$\mathrm{C}_{G}(x)$ is the centralizer of an element $x$ in $G: \mathrm{C}_{G}(x)=\{z \in G \mid z x=x z\}$.

$\mathrm{C}_{G}(M)=\bigcap_{x \in M} \mathrm{C}_{G}(x)$ is the centralizer of a subset $M$ in $G$.

If $A \leq B$ and $A, B \unlhd G$, then $\mathrm{C}_{G}(B / A)=H$, where $H / A=\mathrm{C}_{G / A}(B / A)$. 
$A$ wr $B$ is the wreath product of the 'passive' group $A$ and the transitive permutation group $B$ (in what follows we assume that $B$ is regular); $B$ is called the active factor of the wreath product). Then the order of that group is $|A|^{|B|}|B|$.

$\operatorname{Aut}(G)$ is the group of automorphisms of $G$ (the automorphism group of $G$ ).

$\operatorname{Inn}(G)$ is the group of all inner automorphisms of $G$.

$\operatorname{Out}(G)=\operatorname{Aut}(G) / \operatorname{Inn}(G)$, the outer automorphism group of $G$.

If $a, b \in G$, then $a^{b}=b^{-1} a b$.

An element $x \in G$ inverts a subgroup $H \leq G$ if $h^{x}=h^{-1}$ for all $h \in H$.

If $M \subseteq G$, then $\langle M\rangle=\langle x \mid x \in M\rangle$ is the subgroup of $G$ generated by $M$.

$M^{x}=x^{-1} M x=\left\{y^{x} \mid y \in M\right\}$ for $x \in G$ and $M \subseteq G$.

$[x, y]=x^{-1} y^{-1} x y=x^{-1} x^{y}$ is the commutator of elements $x, y$ of $G$. If $M, N \subseteq G$ then $[M, N]=\langle[x, y] \mid x \in M, y \in N\rangle$ is a subgroup of $G$.

$o(x)$ is the order of an element $x$ of $G$.

An element $x \in G$ is a $\pi$-element if $\pi(o(x)) \subseteq \pi$.

$G$ is a $\pi$-group, if $\pi(G) \subseteq \pi$. Obviously, $G$ is a $\pi$-group if and only if all of its elements are $\pi$-elements.

$G^{\prime}$ is the subgroup generated by all commutators $[x, y], x, y \in G$ (i.e., $G^{\prime}=[G, G]$ ), $G^{(2)}=\left[G^{\prime}, G^{\prime}\right]=G^{\prime \prime}=\left(G^{\prime}\right)^{\prime}, G^{(3)}=\left[G^{\prime \prime}, G^{\prime \prime}\right]=\left(G^{\prime \prime}\right)^{\prime}$ and so on. $G^{\prime}$ is called the commutator (or derived) subgroup of $G$.

$\mathrm{Z}(G)=\bigcap_{x \in G} \mathrm{C}_{G}(x)$ is the center of $G$.

$\mathrm{Z}_{i}(G)$ is the $i$-th member of the upper central series of $G$; in particular, $\mathrm{Z}_{0}(G)=\{1\}$, $\mathrm{Z}_{1}(G)=\mathrm{Z}(G)$.

$\mathrm{K}_{i}(G)$ is the $i$-th member of the lower central series of $G$; in particular, $\mathrm{K}_{2}(G)=G^{\prime}$. We have $\mathrm{K}_{i}(G)=[G, \ldots, G](i \geq 1$ times $)$. We set $\mathrm{K}_{1}(G)=G$.

If $G$ is nonabelian, then $\eta(G) / \mathrm{K}_{3}(G)=\mathrm{Z}\left(G / \mathrm{K}_{3}(G)\right)$.

$\mathcal{M}(G)=\langle x \in G| \mathrm{C}_{G}(x)=\mathrm{C}_{G}\left(x^{p}\right)$ is the Mann subgroup of a $p$-group $G$.

$\operatorname{Syl}_{p}(G)$ is the set of $p$-Sylow subgroups of an arbitrary finite group $G$.

$\mathrm{S}_{n}$ is the symmetric group of degree $n$.

$\mathrm{A}_{n}$ is the alternating group of degree $n$

$\Sigma_{p^{n}}$ is a Sylow $p$-subgroup of $\mathrm{S}_{p^{n}}$.

$\operatorname{GL}(n, F)$ is the set of all nonsingular $n \times n$ matrices with entries in a field $F$, the $n$-dimensional general linear group over $F, \operatorname{SL}(n, F)=\{A \in \operatorname{GL}(n, F) \mid \operatorname{det}(A)=$ $1 \in F\}$, the $n$-dimensional special linear group over $F$. 
If $H \leq G$, then $H_{G}=\bigcap_{x \in G} x^{-1} H x$ is the core of the subgroup $H$ in $G$ and $H^{G}=\bigcap_{H \leq N \unlhd G} N$ is the normal closure or normal hull of $H$ in $G$. Obviously, $H_{G} \unlhd G$.

If $G$ is a $p$-group, then $p^{b(x)}=\left|G: \mathrm{C}_{G}(x)\right| ; b(x)$ is said to be the breadth of $x \in G$, where $G$ is a $p$-group; $b(G)=\max \{b(x) \mid x \in G\}$ is the breadth of $G$.

$\Phi(G)$ is the Frattini subgroup of $G$ (= the intersection of all maximal subgroups of $G$ ), $\Phi(\{1\})=\{1\}, p^{\mathrm{d}(G)}=|G: \Phi(G)|$.

$\Gamma_{i}=\left\{H<G|\Phi(G) \leq H| G:, H \mid=p^{i}\right\}, i=1, \ldots, \mathrm{d}(G)$, where $G>\{1\}$.

If $H<G$, then $\Gamma_{1}(H)$ is the set of all maximal subgroups of $H$.

$\exp (G)$ is the exponent of $G$ (the least common multiple of the orders of elements of $G)$. If $G$ is a $p$-group, then $\exp (G)=\max \{o(x) \mid x \in G\}$.

$\mathrm{k}(G)$ is the number of conjugacy classes of $G$ (= $G$-classes), the class number of $G$.

$K_{x}$ is the $G$-class containing an element $x$ (sometimes we also write $\operatorname{ccl}_{G}(x)$ ).

$\mathrm{C}_{m}$ is the cyclic group of order $m$.

$G^{m}$ is the direct product of $m$ copies of a group $G$.

$A \times B$ is the direct product of groups $A$ and $B$.

$A * B$ is a central product of groups $A$ and $B$, i.e., $A * B=A B$ with $[A, B]=\{1\}$.

$\mathrm{E}_{p^{m}}=\mathrm{C}_{p}^{m}$ is the elementary abelian group of order $p^{m}$. $G$ is an elementary abelian $p$-group if and only if it is a $p$-group $>\{1\}$ and $G$ coincides with its socle. Next, $\{1\}$ is elementary abelian for each prime $p$.

A group $G$ is said to be homocyclic if it is a direct product of isomorphic cyclic subgroups (obviously, elementary abelian $p$-groups are homocyclic).

$\mathrm{ES}(m, p)$ is an extraspecial group of order $p^{1+2 m}$ (a $p$-group $G$ is said to be extraspecial if $G^{\prime}=\Phi(G)=\mathrm{Z}(G)$ is of order $p$ ). Note that for each $m \in \mathbb{N}$, there are exactly two nonisomorphic extraspecial groups of order $p^{2 m+1}$.

$S\left(p^{3}\right)$ is a nonabelian group of order $p^{3}$ and exponent $p>2$.

A special $p$-group is a nonabelian $p$-group $G$ such that $G^{\prime}=\Phi(G)=\mathrm{Z}(G)$ is elementary abelian. Direct products of extraspecial $p$-groups are special.

$\mathrm{D}_{2 m}$ is the dihedral group of order $2 m, m>2$. Some authors consider $\mathrm{E}_{2^{2}}$ as the dihedral group $\mathrm{D}_{4}$.

$\mathrm{Q}_{2^{m}}$ is the generalized quaternion group of order $2^{m} \geq 2^{3}$.

$\mathrm{SD}_{2^{m}}$ is the semidihedral group of order $2^{m} \geq 2^{4}$.

$\mathbf{M}_{p^{m}}$ is a nonabelian $p$-group containing exactly $p$ cyclic subgroups of index $p$. 
$\operatorname{cl}(G)$ is the nilpotence class of a $p$-group $G$.

$\operatorname{dl}(G)$ is the derived length of a $p$-group $G$.

$\mathrm{CL}(G)$ is the set of all $G$-classes.

A $p$-group of maximal class is a nonabelian group $G$ of order $p^{m}$ with $\operatorname{cl}(G)=m-1$. $\Omega_{m}(G)=\left\langle x \in G \mid o(x) \leq p^{m}\right\rangle, \Omega_{m}^{*}(G)=\left\langle x \in G \mid o(x)=p^{m}\right\rangle$ and $\mho_{m}(G)=$ $\left\langle x^{p^{m}} \mid x \in G\right\rangle$.

A $p$-group is absolutely regular if $\left|G / \mho_{1}(G)\right|<p^{p}$.

A $p$-group is thin if it is either absolutely regular or of maximal class.

$G=A \cdot B$ is a semidirect product with kernel $B$ and complement $A$.

A group $G$ is an extension of $N \unlhd G$ by a group $H$ if $G / N \cong H$. A group $G$ splits over $N$ if $G=H \cdot N$ with $H \leq G$ and $H \cap N=\{1\}$ (in that case, $G$ is a semidirect product of $H$ and $N$ with kernel $N$ ).

$H^{\#}=H-\left\{e_{H}\right\}$, where $e_{H}$ is the identity element of the group $H$. If $M \subseteq G$, then $M^{\#}=M-\left\{e_{G}\right\}$.

An automorphism $\alpha$ of $G$ is regular (= fixed-point-free) if it induces a regular permutation on $G^{\#}$ (a permutation is said to be regular if it has no fixed points).

An involution is an element of order 2 in a group.

A section of a group $G$ is an epimorphic image of some subgroup of $G$.

If $F=\operatorname{GF}\left(p^{n}\right)$, then we write $\operatorname{GL}\left(m, p^{n}\right), \operatorname{SL}\left(m, p^{n}\right), \ldots$ instead of $\operatorname{GL}(m, F)$, $\mathrm{SL}(m, F), \ldots$.

$\mathrm{c}_{n}(G)$ is the number of cyclic subgroups of order $p^{n}$ in a $p$-group $G$.

$\mathrm{s}_{n}(G)$ is the number of subgroups of order $p^{n}$ in a $p$-group $G$.

$e_{n}(G)$ is the number of subgroups of order $p^{n}$ and exponent $p$ in $G$.

$\mathcal{A}_{n}$-group is a $p$-group $G$ all of whose subgroups of index $p^{n}$ are abelian but $G$ contains a nonabelian subgroup of index $p^{n-1}$. In particular, $\mathcal{A}_{1}$-group is a minimal nonabelian $p$-group for some $p$.

$\alpha_{n}(G)$ is the number of $\mathcal{A}_{n}$-subgroups in a $p$-group $G$.

\section{Characters and representations}

$\operatorname{Irr}(G)$ is the set of all irreducible characters of $G$ over $\mathbb{C}$.

A character of degree 1 is said to be linear.

$\operatorname{Lin}(G)$ is the set of all linear characters of $G$ (obviously, $\operatorname{Lin}(G) \subseteq \operatorname{Irr}(G)$ ). 
$\operatorname{Irr}_{1}(G)=\operatorname{Irr}(G)-\operatorname{Lin}(G)$ is the set of all nonlinear irreducible characters of $G$; $\mathrm{n}(G)=\left|\operatorname{Irr}_{1}(G)\right|$.

$\chi(1)$ is the degree of a character $\chi$ of $G$,

$\chi_{H}$ is the restriction of a character $\chi$ of $G$ to $H \leq G$.

$\chi^{G}$ is the character of $G$ induced from the character $\chi$ of some subgroup of $G$.

$\bar{\chi}$ is a character of $G$ defined as follows: $\bar{\chi}(x)=\overline{\chi(x)}$ (here $\bar{w}$ is the complex conjugate of $w \in \mathbb{C}$ ).

$\operatorname{Irr}(\chi)$ is the set of irreducible constituents of a character $\chi$ of $G$.

If $\chi$ is a character of $G$, then $\operatorname{ker}(\chi)=\{x \in G \mid \chi(x)=\chi(1)\}$ is the kernel of a character $\chi$.

$\mathrm{Z}(\chi)=\{x \in G|| \chi(x) \mid=\chi(1)\}$ is the quasikernel of $\chi$.

If $N \unlhd G$, then $\operatorname{Irr}(G \mid N)=\{\chi \in \operatorname{Irr}(G) \mid N \not \leq \operatorname{ker}(\chi)\}$.

$\langle\chi, \tau\rangle=|G|^{-1} \sum_{x \in G} \chi(x) \tau\left(x^{-1}\right)$ is the inner product of characters $\chi$ and $\tau$ of $G$.

$\mathrm{I}_{G}(\phi)=\left\langle x \in G \mid \phi^{x}=\phi\right\rangle$ is the inertia subgroup of $\phi \in \operatorname{Irr}(H)$ in $G$, where $H \triangleleft G$.

$1_{G}$ is the principal character of $G\left(1_{G}(x)=1\right.$ for all $\left.x \in G\right)$.

$\mathrm{M}(G)$ is the Schur multiplier of $G$.

$\operatorname{cd}(G)=\{\chi(1) \mid \chi \in \operatorname{Irr}(G)\}$.

$\operatorname{mc}(G)=\mathrm{k}(G) /|G|$ is the measure of commutativity of $G$.

$\mathrm{T}(G)=\sum_{\chi \in \operatorname{Irr}(G)} \chi(1), \mathrm{f}(G)=\mathrm{T}(G) /|G|$. 